\title{
Relation between chiral central charge and ground-state degeneracy in $(2+1)$-dimensional topological orders
}

\author{
Liang Kong ${ }^{1}$ and Xiao-Gang Wen ${ }^{2}$ \\ ${ }^{1}$ Guangdong Provincial Key Laboratory of Quantum Science and Engineering and Shenzhen Institute for Quantum Science and Engineering, \\ Southern University of Science and Technology, Shenzhen 518055, China \\ ${ }^{2}$ Department of Physics, Massachusetts Institute of Technology, Cambridge, Massachusetts 02139, USA
}

(Received 25 April 2020; revised 17 June 2020; accepted 14 July 2020; published 1 September 2020)

\begin{abstract}
A bosonic topological order on $d$-dimensional closed space $\Sigma^{d}$ may have degenerate ground states. The space $\Sigma^{d}$ with different shapes (different metrics) form a moduli space $\mathcal{M}_{\Sigma^{d}}$. Thus the degenerate ground states on every point in the moduli space $\mathcal{M}_{\Sigma^{d}}$ form a complex vector bundle over $\mathcal{M}_{\Sigma^{d}}$. It has been suggested that the collection of such vector bundles for $d$-dimensional closed spaces of all topologies completely characterizes the topological order. Using such a point of view, we propose a direct relation between two seemingly unrelated properties of (2+1)-dimensional topological orders: (i) the chiral central charge $c$ that describes the many-body density of states for edge excitations (or more precisely the thermal Hall conductance of the edge) and (ii) the ground-state degeneracy $D_{g}$ on the closed genus- $g$ surface. We show that $c D_{g} / 2 \in \mathbb{Z}, g \geqslant 3$, for bosonic topological orders. We explicitly check the validity of this relation for over 140 simple topological orders. For fermionic topological orders, we let $D_{g, \sigma}^{e}\left(D_{g, \sigma}^{o}\right)$ be the degeneracy with an even (odd) number of fermions on the genus- $g$ surface with spin structure $\sigma$. Then we have $2 c D_{g, \sigma}^{e} \in \mathbb{Z}$ and $2 c D_{g, \sigma}^{o} \in \mathbb{Z}$ for $g \geqslant 3$.
\end{abstract}

DOI: 10.1103/PhysRevResearch.2.033344

\section{INTRODUCTION}

Through a theoretical study of chiral spin liquid [1,2], we realized that there exists a new kind of order, topological order [3-5], beyond Landau symmetry-breaking theory. Topological order cannot be characterized by the local order parameters associated with the symmetry breaking. However, topological order can be characterized or defined by the following macroscopic properties: (i) the topology-dependent ground-state degeneracy $[3,4]$, and (ii) the non-Abelian geometric phases of the degenerate ground states as we deform the metrics of the space [5,6]. Both of the above macroscopic properties are robust against any local perturbations that can break any symmetries [4]. This is just like superfluid order, which is characterized or defined by zero viscosity and quantized vorticity that are robust against any local perturbations that preserve the U(1) symmetry.

For topological orders in $(2+1)$-dimensional $(2+1 \mathrm{D})$ space-time, the non-Abelian geometric phases of the degenerate ground states encode the chiral central charge $c$ of the topological order via the gravitational Chern-Simons term in the effective action [7-9]. (The edge of a topological order contains right movers with central charge $c_{R}$, and left movers with central charge $c_{L}$. The chiral central charge $c=c_{R}-c_{L}$ $[10,11])$. In this paper we propose a direct relation between

Published by the American Physical Society under the terms of the Creative Commons Attribution 4.0 International license. Further distribution of this work must maintain attribution to the author(s) and the published article's title, journal citation, and DOI. the ground-state degeneracy $D_{g}$ on a genus- $g$ space and the chiral central charge $c$ of the edge states, for 2+1D topological orders in bosonic systems:

$$
\frac{1}{2} c D_{g} \in \mathbb{Z} \quad \text { for } g \geqslant 3 \text {. }
$$

For $2+1 \mathrm{D}$ topological orders in fermionic systems, we propose

$$
2 c D_{g, \sigma}^{e} \in \mathbb{Z}, \quad 2 c D_{g, \sigma}^{e} \in \mathbb{Z} \quad \text { for } g \geqslant 3,
$$

where $D_{g, \sigma}^{e}\left(D_{g, \sigma}^{o}\right)$ is the degeneracy with even (odd) number of fermions on genus- $g$ surface with spin structure $\sigma$. This result can be derived from the characterization of a topological order in terms of a complex vector bundle on the moduli space $\mathcal{M}_{\Sigma^{d}}$ of a closed space $\Sigma^{d}$, where the fiber is the degenerate ground states on $\Sigma^{d}$. For an introduction on vector bundle, as well as Chern and Pontryagin classes, see Ref. [12]. In the Appendix we also discuss how to use the partition function on arbitrary closed space-time $M^{D}$ and the resulting complex function on $\mathcal{M}_{M^{D}}$, the moduli space of $M^{D}$, to characterize topological orders.

In this paper, we will use $d$ or $1 d, 2 d$, etc., to denote the space dimension and $D$ or $1+1 \mathrm{D}, 2+1 \mathrm{D}$, etc., to denote the space-time dimension. We will only consider anomaly-free topological orders, i.e., the topological orders that can be realized by local lattice models in the same dimension. In contrast, an anomalous topological order can only be realized as a boundary of the local lattice model in one higher dimension $[13,14]$. 


\section{PROBING AND MEASURING THE TOPOLOGICAL ORDERS: THE HAMILTONIAN APPROACH}

The main issue that we are going to discuss in this paper is how to probe and measure different topological orders using macroscopic properties. Here "probe and measure" means the methods in experiments and/or numerical calculations that allow us to distinguish different topological orders.

\section{A. Complex vector bundle on the moduli space of the space manifold}

In Hamiltonian approach, an anomaly-free topological order is described by a local bosonic Hamiltonian acting on a Hilbert space $\mathcal{V}_{\text {tot }}$ with a tensor product decomposition

$$
\mathcal{V}_{\mathrm{tot}}=\bigotimes_{i} \mathcal{V}_{i}
$$

where $\mathcal{V}_{i}$ is the finite-dimensional Hilbert space for site $i$. The Hamiltonian is required to be well defined for arbitrary space $\Sigma^{d}$ with arbitrary triangulation. Since anomaly-free topological orders are gapped, we require the Hamiltonian on a closed space $\Sigma^{d}$ to be gapped, whose degenerate ground states form a finite-dimensional vector space $\mathcal{V}_{\text {grnd }}$, which is a subspace of the total Hilbert space $\mathcal{V}_{\text {tot }}$ of the boson system. Let $\mathcal{M}_{\Sigma^{d}}$ be the moduli space for closed space $\Sigma^{d}$ with different metrics and $\mathcal{M}$ the disjoint union of these moduli spaces. We see that there is a ground-state vector space $\mathcal{V}_{\text {grnd }}$ for every point in $\mathcal{M}_{\Sigma^{d}}$. Therefore, for each $\Sigma^{d}$, an anomaly-free topological order gives rise to a complex vector bundle $\mathcal{E}_{\Sigma^{d}}$ with fiber $\mathcal{V}_{\text {grnd }}$ and base space $\mathcal{M}_{\Sigma^{d}}$. It was proposed in Ref. [5] that:

Claim 1. The complex vector bundle

$$
p t \rightarrow \mathcal{V}_{\text {grnd }} \rightarrow \mathcal{E}_{\Sigma^{d}} \rightarrow \mathcal{M}_{\Sigma^{d}} \rightarrow p t
$$

of degenerate ground states on $\mathcal{M}_{\Sigma^{d}}$ (for all $\Sigma^{d}$ 's) fully characterize an anomaly-free topological order in Hamiltonian formalism.

To understand the vector bundle $\mathcal{E}_{\Sigma^{d}}$, let us use $G_{\text {homeo }}\left(\Sigma^{d}\right)$ to denote the orientation preserving homeomorphism group of the space $\Sigma^{d}$. Note that $G_{\text {homeo }}\left(\Sigma^{d}\right)$ only depends on the topology of $\Sigma^{d}$ and is the same for every point $\Sigma^{d} \in \mathcal{M}_{\Sigma^{d}}$. Let us use $G_{\text {homeo }}^{0}\left(\Sigma^{d}\right)$ to denote the subgroup of $G_{\text {homeo }}\left(\Sigma^{d}\right)$, which is the connected component of $G_{\text {homeo }}\left(\Sigma^{d}\right)$ that contain identity. The mapping class group is formed by the discrete components of the homeomorphism group:

Definition 1. Mapping class group $\operatorname{MCG}\left(\Sigma^{d}\right) \equiv$ $G_{\text {homeo }}\left(\Sigma^{d}\right) / G_{\text {homeo }}^{0}\left(\Sigma^{d}\right)=\pi_{0}\left[G_{\text {homeo }}\left(\Sigma^{d}\right)\right]$.

We note that every homeomorphism $g: \Sigma^{d} \rightarrow \Sigma^{d}$ in $\operatorname{MCG}\left(\Sigma^{d}\right)$ defines a mapping torus $\Sigma^{d} \curlywedge_{g} S^{1}$ that describes how $\Sigma^{d}$ deform around a loop $S^{1}$, and corresponds to an element in $\pi_{1}\left(\mathcal{M}_{\Sigma^{d}}\right)$. Thus $\pi_{1}\left(\mathcal{M}_{\Sigma^{d}}\right)=\operatorname{MCG}\left(\Sigma^{d}\right)$.

Along a loop $g$ in $\pi_{1}\left(\mathcal{M}_{\Sigma^{d}}\right)$, the fiber bundle gives us a monodromy $U(g)$, which is a unitary matrix acting on the ground-state vector space $\mathcal{V}_{\text {grnd }}$. We may view $g$ as an element in the group $\operatorname{MCG}\left(\Sigma^{d}\right)$. So $U(g)$ gives a projective representation of $\operatorname{MCG}\left(\Sigma^{d}\right)$ [7].

To understand why we only get a projective representation, we note that the topological robustness of the ground-state degeneracy implies that the unitary matrix $U$ (id) for a contractible loop $g=\mathrm{id}$ in the moduli space $\mathcal{M}_{\Sigma^{d}}$ must be a pure overall phase (which can be path dependent), so that $U$ (id) cannot distinguish (or split) the degenerate ground states. This is because the periodic time evolution along a contractable loop over and over again can be simulated by a local Hamiltonian. If $U$ (id) can distinguish the degenerate ground states, then there is a local Hamiltonian that can distinguish and split the degenerate ground states. This contradicts with the fact that the topological degeneracy of ground states cannot be lifted by any local Hamiltonian. Similarly, $U(g)$ may also depend on paths, but the path-dependent part must be an overall phase. This leads to the projective representation of $\operatorname{MCG}\left(\Sigma^{d}\right)$. We also like to mention that the trace of $U(g)$ is the volume-independent partition function (see Appendix 1 for details) on the corresponding mapping torus:

$$
\operatorname{Tr} U(g)=Z^{\mathrm{top}}\left(\Sigma^{d} \curlywedge_{g} S^{1}\right) .
$$

As a result, we obtain

$$
\left|Z^{\text {top }}\left(\Sigma^{d} \times S^{1}\right)\right|=\text { ground-state degeneracy on } \Sigma^{d} .
$$

For spaces with different topologies, we get different projective representations. Those finite-dimensional projective representations are the non-Abelian geometric phases of the degenerate ground states introduced in Refs. [5,6]. Certainly, the non-Abelian geometric phases contain more information than the projective representations. They contain all the information about the vector bundle $\mathcal{E}_{\Sigma^{d}}$ on $\mathcal{M}_{\Sigma^{d}}$. Therefore, we believe that such geometric phases for closed $d$ dimensional spaces $\Sigma^{d}$ of all topologies fully characterize the topological order.

We would like to remark that for a generic system, its vector bundle $\mathcal{E}_{\Sigma^{d}}$ is generally not flat. The curvature of the vector bundle can change as we deform the Hamiltonian locally. However, for some topological orders, its vector bundle $\mathcal{E}_{\Sigma^{d}}$ cannot be made flat no matter how we fine tune the Hamiltonian. In this case, the vector bundle $\mathcal{E}_{\Sigma^{d}}$ is topologically different from a flat bundle. In this case, the volume-independent partition function on mapping torus $Z^{\text {top }}\left(\Sigma^{d} \lambda_{g} S^{1}\right)$ cannot be topological (i.e., the volume-independent partition function cannot be a constant on a connected piece of the moduli space $\mathcal{M}_{\Sigma^{d}}$ ). It must depend on the metrics of the space-time $\Sigma^{d} \curlywedge_{g} S^{1}$.

It is very strange since the bosonic system has short-range correlation and a finite energy gap. In the thermodynamical limit, the space-time becomes flat, and the bosonic system should not be able to sense the geometry of the space-time. The fact that the partition function does depend on the metrics of the space-time means that the entanglement in the ground state can still sense the geometry of the space in the flat limit. We like to link such a geometric sensitivity to the gapless nature of boundary excitations and entanglement spectrum:

Claim 2. The ground-state vector bundle $\mathcal{E}_{\Sigma^{d}}$ over $\mathcal{M}_{\Sigma^{d}}$ can be deformed into a flat bundle if and only if the boundary of the corresponding anomaly-free topological order is gappable.

What is the obstruction that prevents the vector bundle to be flat? First, for a contractible loop $g=\mathrm{id}, U$ (id) is a pure $U(1)$ phase. So the nonflat part is only contained in the $U(1)$ phase of the complex vector bundle. We can examine it by considering the determinant line bundle $\mathcal{E}_{\Sigma^{d}}^{\text {det }}$ of the vector bundle $\mathcal{E}_{\Sigma^{d}}$, which is a complex line bundle over $\mathcal{M}_{\Sigma^{d}}$. 
To connect the determinant line bundle to the partition fuction of the system, let us consider a contractable loop $S^{1}$ in $\mathcal{M}_{\Sigma^{d}}$. We have mentioned that the partition function for the space-time $\Sigma^{d} \times S^{1}$ is given by the monodromy $U$ (id) $=$ $e^{i \theta}$ along the loop [see Eq. (5) and remember that $U$ (id) is pure phase factor]. Therefore the partition function of the system on space-time $\Sigma^{d} \times S^{1}$ [see Eq. (13) for an example] is given by

$$
\begin{aligned}
Z^{\mathrm{top}}\left(\Sigma^{d} \times S^{1}\right) & =D_{\Sigma^{d}} e^{i \theta} \\
\text { or }\left[Z^{\mathrm{top}}\left(\Sigma^{d} \times S^{1}\right)\right]^{D_{\Sigma^{d}}} & =\left(D_{\Sigma^{d}}\right)^{D_{\Sigma^{d}} \operatorname{Det} U(\mathrm{id}),}
\end{aligned}
$$

where $D_{\Sigma^{d}}=\operatorname{Dim} U$ (id) is the ground-state degeneracy on the closed space $\Sigma^{d}$, and $\operatorname{Det} U$ (id) is the monodromy of the determinant line bundle around the loop $S^{1}$. Now, let us assume that the loop $S^{1}$ is the boundary of a two-dimension submanifold $B \subset \mathcal{M}_{\Sigma^{d}}$ : $S^{1}=\partial B$. We can rewrite that above as [for the $U(1)$ phase factor]

$$
\begin{aligned}
{\left[Z^{\mathrm{top}}\left(\Sigma^{d} \times S^{1}\right)\right]^{D_{\Sigma^{d}}} } & \propto \operatorname{Det} U(\mathrm{id})=e^{i 2 \pi \int_{B} C} \\
& =e^{i 2 \pi D_{\Sigma^{d}} \int_{\Sigma^{d} \curlywedge B} P},
\end{aligned}
$$

where $C$ is the curvature tensor on the moduli space $\mathcal{M}_{\Sigma^{d}}$ for the determinant line bundle. The next expression $e^{i 2 \pi D_{\Sigma^{d}} \int_{\Sigma^{d}<B} P}$ is motivated by noticing that $Z^{\text {top }}\left(\Sigma^{d} \times S^{1}\right)$ is given by a gravitational Chern-Simon term $\omega$

$$
Z^{\mathrm{top}}\left(\Sigma^{d} \times S^{1}\right) \propto e^{i 2 \pi \int_{\Sigma^{d} \times S^{1}} \omega} .
$$

In Appendix 1 we discussed how to define the volumeindependent partition function $Z^{\text {top }}$. A key character of $Z^{\text {top }}$ is that it is invariant under the scaling transformation of space-time $(t, x, y, \cdots) \rightarrow(c t, c x, c y, \cdots)$. Since the lowenergy effective action for gapped liquid state is local and does not contain operators like $\frac{\partial_{x}}{\partial_{y}}$, the above isotropic scale invariance implies an invariance of $Z^{\text {top }}$ under a more general nonisotropic scaling transformation $(t, x, y, \cdots) \rightarrow$ $\left(c_{t} t, c_{x} x, c_{y} y, \cdots\right)$. The gravitational Chern-Simon term $\omega$ is believed to be the only term that is invariant under the nonisotropic scaling transformation, and at the same time depends on the space-time metrics. There are other topological terms that are invariant under the nonisotropic scaling transformation. But those terms are invariant under any local deformation of the space-time metrics. Here we ignore such local constant terms, because they do not contribute to $\int_{\Sigma^{d} \curlywedge B} P$ in Eq. (8). In $2+1$ dimensions, some explicit calculations have been done [7-9], which confirm the above picture.

We can rewrite Eq. (9) in term of a linear combination of Pontryagin class on $\Sigma^{d}<B$

$$
Z^{\mathrm{top}}\left(\Sigma^{d} \times S^{1}\right) \propto e^{i 2 \pi \int_{\Sigma^{d} \times S^{1}} \omega}=e^{i 2 \pi \int_{\Sigma^{d} \curlywedge B} P}
$$

since $\partial\left(\Sigma^{d} \wedge B\right)=\Sigma^{d} \times S^{1}$. Here $\Sigma^{d} \wedge B$ is a fiber bundle with the space $\Sigma^{d}$ as the fiber and $B$ as the base manifold. Also $P=d \omega$ is a linear combination of Pontryagin class on $\Sigma^{d} \wedge B$ :

$$
\begin{aligned}
P & =\sum_{n_{1}, n_{2}, \cdots} \kappa_{n_{1} n_{2} \ldots} P_{n_{1} n_{2} \ldots} \\
P_{n_{1} n_{2} \cdots} & =p_{n_{1}} p_{n_{2}} \cdots, \quad \kappa_{n_{1} n_{2} \ldots} \ldots \mathcal{Q},
\end{aligned}
$$

and $p_{n}$ is the $n$th Pontryagin class. This leads to the expression Eq. (8). This is a key assumption in this paper.

Now, let us shrink the loop $S^{1}$ to a point and $B$ becomes a closed two-dimensional submanifold in $\mathcal{M}_{\Sigma^{d}}$. Then, $\int_{B} C$ becomes the Chern number of the line bundle $\mathcal{E}_{\Sigma^{d}}^{\text {det }}$ on $B$, which always is an integer. We obtain

$$
D_{\Sigma^{d}} \int_{\Sigma^{d} \curlywedge B} P=\text { integer. }
$$

This expression gives us a constraint between the ground-state degeneracy $D_{\Sigma^{d}}$ and the gravitational Chern-Simons term in the effective theory. It is the main result of this paper. We remark that the above result is obtained with an assumption that the ground states of topological order can be put the closed space $\Sigma^{d}$ without the need to create some topological excitations. Otherwise, the above can still be valid if we set $D_{\Sigma^{d}}=0$ when we have to create topological excitations. We would also like to point out the first Chern class $C$ (i.e., the collection Chern numbers $\int_{B} C$ for all closed two-dimensional subspaces of $\mathcal{M}_{\Sigma^{d}}$ ) completely classify the line bundle $\mathcal{E}_{\Sigma^{d}}^{\mathrm{det}}$.

Let us consider an example of $2 d$ theory whose gravitational response contains the gravitational Chern-Simons term [7-9]:

$$
Z^{\text {top }}\left(\Sigma^{2}<S^{1}\right)=e^{i \frac{2 \pi c}{24} \int_{\Sigma^{2} \curlywedge S^{1}} \omega_{3}}, \quad d \omega_{3}=p_{1},
$$

where $c$ is the chiral central charge of the edge states. For such a theory, Eq. (12) becomes

$$
\frac{c}{24} D_{g} \int_{\Sigma^{2} \curlywedge B^{2}} p_{1}=\text { integer, }
$$

for any surface bundle $\Sigma^{2} \wedge B^{2}$, where $D_{g}$ is the ground-state degeneracy on $\Sigma^{2}$, and $g$ is the genus of $\Sigma^{2}$.

Since $\int_{\Sigma^{2}\left\langle B^{2}\right.} p_{1} \neq 0$ for some surface bundle, $\int_{B^{2}} C \neq 0$ for some $B$ and the vector bundle $\mathcal{E}_{\Sigma^{d}}$ is not flat if $c \neq 0$. So the appearance of the gravitational Chern-Simons term implies that the vector bundle $\mathcal{E}_{\Sigma^{d}}$ is not flat.

It was shown that $\int_{\Sigma^{2} \curlywedge B^{2}} p_{1}=0 \bmod 12$ for any orientable surface bundles $[15,16]$. If the genus of the fiber $\Sigma^{2}$ is equal or less than 2, then $\int_{\Sigma^{2} \wedge B^{2}} p_{1}=0[15,17,18]$. If the genus of the fiber $\Sigma^{2}$ is equal or greater than 3 , then we can always find a base manifold $B^{2}$ with a genus equal or less than 111 , such that there is a surface bundle $\Sigma^{2} \curlywedge B^{2}$ with $\int_{\Sigma^{2} \curlywedge B^{2}} p_{1}= \pm 12$ [18]. Therefore: ${ }^{1}$

Claim 3. For a $2 d$ bosonic topological orders, the chiral central charge of the edge state is quantized as $c D_{g} / 2 \in \mathcal{Z}$ for $g \geqslant 3$, where $D_{g}$ is the ground-state degeneracy on genus- $g$ space.

The above result implies that the chiral central charge $c$ is a rational number, which was proven via some other methods $[19,20]$. Let us give some nontrivial checks for Claim 3.

Application 1. For a bosonic quantum Hall state with one branch of edge mode (i.e., $c=1$ ), the ground-state degeneracy $D_{g}$ must be even for $g \geqslant 3$.

${ }^{1}$ Claim 3 was first obtained in our long unpublished paper Ref. [14]. This paper simplifies and extends the result to get it published. 
Application 2. For a $2 d$ bosonic topological order, we can use $i, j, k$ to label the topological excitations. The fusion rule is given by $i \otimes j=\oplus_{k} N_{i j}^{k} k$. The ground-state degeneracy $D_{g}$ is then given by [21]

$$
\begin{aligned}
D_{g} & =\operatorname{Tr}\left(\sum_{i} N_{i} N_{\bar{i}}\right)^{g-1}=\sum_{i} S_{1 i}^{-2(g-1)} \\
& =\left(\sum_{i} d_{i}^{2}\right)^{g-1} \sum_{i} d_{i}^{-2(g-1)},
\end{aligned}
$$

where $\bar{i}$ is the antiparticle of $i$, the matrix $N_{i}$ is given by $\left(N_{i}\right)_{j}^{k}=N_{i j}^{k}$, and $d_{i}$ is the quantum dimension (the largest eigenvalue of $N_{i}$ ). Also $S_{i j}$ is the matrix elements of the $S$ matrix that characterizes the topological order $[5,6]$. We have $S_{1 i}=\frac{d_{i}}{\sqrt{\sum_{i} d_{i}^{2}}}$.

For filling fraction $v=1$ bosonic Pfaffian quantum Hall state, we have $\left(d_{i}\right)=(1,1, \sqrt{2})$. We find that $D_{1}=3, D_{2}=$ $10, D_{3}=36, D_{4}=136, D_{5}=528$, etc. Therefore the chiral central charge must be quantized as $c=0 \bmod 1 / 2$, which agrees with $c=3 / 2$. We also see that $c D_{g} / 2=$ integer is not valid for $g=2$.

For the Fibonacci topological order with $\left(d_{i}\right)=\left(1, \frac{\sqrt{5}+1}{2}\right)$ and $c=14 / 5$, we find that $D_{1}=2, D_{2}=5, D_{3}=15, D_{4}=$ $50, D_{5}=175$, etc. Indeed, $c D_{g} / 2=$ integer for $g \geqslant 3$. We explicitly checked over 140 simple topological orders listed in Ref. [22] and find that Claim 3 is valid for those bosonic topological orders.

Application 3. The chiral central charge of $2 d$ invertible anomaly-free topological order is quantized as $c=0 \bmod 2$, since $D_{g}=1$. A known $2 d$ invertible anomaly-free topological order is the $E_{8}$ state, which has $c=8$. At the moment, we do not know if the minimal chiral central charge $c=2$ can be realized by a $2 d$ invertible anomaly-free topological order.

If we have a fermionic system, both $\Sigma^{d}$ and $\Sigma^{d} \curlywedge B^{2}$ should be chosen to be spin manifolds. In this case $\Sigma^{d}$ can have a spin structure, denoted as $\sigma$, which can be extended to $\Sigma^{d}\left\langle B^{2}\right.$. The ground states on $\Sigma^{d}$ can carry even or odd numbers of fermions. We denote the ground-state degeneracy with even fermions as $D_{\Sigma^{d}, \sigma}^{e}$ and that with odd fermions as $D_{\Sigma^{d}, \sigma}^{o}$. We note that the even and odd sectors do not mix due to the conservation of fermion number parity. Therefore, we have two vector bundles on the modular space $\mathcal{M}_{\Sigma^{d}}$.

In two-dimensional space $(d=2)$, when $\Sigma^{2} \curlywedge B^{2}$ is spin, we have $\int_{\Sigma^{2} \measuredangle B^{2}} p_{1}=0$ mod 48 for any spin surface bundles $[15,17]$. Assuming that $\int_{\Sigma^{2}\left\langle B^{2}\right.} p_{1}= \pm 48$ can be realized for some surface bundle $\Sigma^{2}\left\langle B^{2}\right.$ if the genus of $\Sigma^{2}$ is greater than 2, we find that:

Claim 4. For fermionic topological orders, the chiral central charge is quantized as

$$
2 c D_{g, \sigma}^{e} \in \mathcal{Z}, \quad 2 c D_{g, \sigma}^{o} \in \mathcal{Z}, \quad g \geqslant 3,
$$

where $D_{g, \sigma}^{e}\left(D_{g, \sigma}^{e}\right)$ is the ground-state degeneracy on closed genus- $g$ surface with spin structure $\sigma$ and even (odd) number of fermions.

For fermionic invertible topological orders, we have $D_{g, \sigma}^{e}+D_{g, \sigma}^{o}=1$ and the chiral central charge is quantized as $c=0$ mod $1 / 2$. The minimal chiral central charge $c=1 / 2$ for fermionic invertible topological orders can be realized by $p+i p$ superconductor, which indeed contain no nontrivial topological excitations.

For a $2 d$ fermionic topological order, the quantum dimensions of excitations appear pairs of equal values: $d_{2 i}=$ $d_{2 i+1}$ [23]. Many $2 d$ fermionic topological orders are stacking of a fermionic trivial product state and bosonic topological orders with quantum dimensions $d_{i}^{B}$. In this case, we either have $D_{g, \sigma}^{e} \neq 0, D_{g, \sigma}^{o}=0$ or $D_{g, \sigma}^{e}=0, D_{g, \sigma}^{o} \neq 0$. The total ground-state degeneracy $D_{g, \sigma}=D_{g, \sigma}^{e}+D_{g, \sigma}^{o}$ is indenpendent of spin structure. To compute $D_{g, \sigma}$, we note that the quantum dimensions for the resulting fermion topological order are given by $d_{2 i}=d_{2 i+1}=d_{i}^{B}$, and the ground-state degeneracies are the same as the corresponding bosonic topological order: $D_{g, \sigma}=D_{g, \sigma}^{e}+D_{g, \sigma}^{o}=\left(\sum_{i}\left(d_{i}^{B}\right)^{2}\right)^{g-1} \sum_{i}\left(d_{i}^{B}\right)^{-2(g-1)}$, and we obtain

$$
D_{g, \sigma}=D_{g, \sigma}^{e}+D_{g, \sigma}^{o}=\left(\frac{1}{2} \sum_{i} d_{i}^{2}\right)^{g-1} \frac{1}{2} \sum_{i} d_{i}^{-2(g-1)} .
$$

Amazingly, when we apply the above formula to more general fermionic topological orders obtained in Ref. [23], the above expression always give us integers, which satisfy $2 c D_{g, \sigma} \in \mathcal{Z}$ for $g \geqslant 3$.

We like to remark that for fermionic topological orders, $D_{\Sigma^{d}, \sigma}^{e}$ and $D_{\Sigma^{d}, \sigma}^{o}$ may depend on spin structure $\sigma$ (see Refs. [24,25]). It is not clear whether $D_{\Sigma^{d}, \sigma}^{e}+D_{\Sigma^{d}, \sigma}^{o}$ depends on spin structure or not. For the examples examined in Refs. [24,25], $D_{\Sigma^{d}, \sigma}^{e}+D_{\Sigma^{d}, \sigma}^{o}$ does not depend on spin structure.

\section{B. No nontrivial bosonic topological order in $1 d$ space}

Next let us consider bosonic $1 d$ topological orders. Since $\operatorname{MCG}\left(S^{1}\right)$ is trivial, $\mathcal{M}_{\Sigma^{1}}$ is simply connected. Since the Pontryagin classes for circle bundle $S^{1} \wedge B$ all vanishes, the determinant bundle of the vector bundle $\mathcal{E}_{\Sigma^{1}}^{\text {det }}$ over $\mathcal{M}_{\Sigma^{1}}$ can always be deformed into a flat one. Thus the vector bundle $\mathcal{E}_{\Sigma^{1}}$ can be flat. Such a vector bundle is always trivial since $\mathcal{M}_{\Sigma^{1}}$ is simply connected. Therefore, all bosonic anomaly-free $1 d$ topological orders are trivial.

It appears that the vector bundle $\mathcal{E}_{\Sigma^{d}}$ on $\mathcal{M}_{\Sigma^{d}}$ is a highresolution characterization of the anomaly-free topological order. The nontrivial anomaly-free topological order should lead to a nontrivial vector bundle $\mathcal{E}_{\Sigma^{d}}$. On the other hand, since the structure of the vector bundle can be so rich, it is very likely that not every allowed vector bundle $\mathcal{E}_{\Sigma^{d}}$ on $\mathcal{M}_{\Sigma^{d}}$ can be realized by anomaly-free topological orders.

\section{How to probe and measure the boundary-gappable topological orders}

For a boundary-gappable topological order, the vector bundle on $\mathcal{M}_{\Sigma^{d}}$ can always be deformed into a flat one. In fact, the boundary-gappable topological orders can be realized by renormalization-group fixed-point Hamiltonians, which are formed by commuting projectors [26], or by renormalizationgroup fixed-point Lagrangians, which are retriangulation invariant [27,28]. The vector bundles on $\mathcal{M}_{\Sigma^{d}}$ obtained from those fixed-point systems are always flat, and the partition functions on mapping torus are always topological (which are the state-sum topological invariants $[27,28]$ ). For a flat vec- 
tor bundle, the unitary matrices $U(g)$ (the monodromies for noncontractible loops) form a representation (instead of a projective representation) of the mapping class group $\operatorname{MCG}\left(\Sigma^{d}\right)$, which fully characterize the flat bundle [5].

Claim 5. A boundary-gappable topological order is fully characterized by a collection of representations of the mapping class groups $\operatorname{MCG}\left(\Sigma^{d}\right)$ for various spatial topologies.

In particular, the representations of $\operatorname{MCG}\left(\Sigma^{d}\right)$ can be computed via the universal wave function overlap [29-31] or tensor network calculations [32-35].

For $2 d$ boundary-gappable topological orders, the representations of the mapping class group $\operatorname{MCG}\left(\Sigma_{1}\right)$ for genus-1 torus are called the modular data, which already carry a lot of information about the topological orders $[6,22,36]$. However, in Ref. [37] Mignard and Schauenburg found some different topological orders that have the same modular data and chiral central charge. Thus, modular data and chiral central charge are not enough to fully characterize topological order. In Ref. [38] its was shown that if we include the representations of the mapping class group $\operatorname{MCG}\left(\Sigma_{2}\right)$ for genus-2 surface, then those topological orders can be distinguished. This supports the conjecture proposed in Ref. [5] that the representations of the mapping class groups $\operatorname{MCG}\left(\Sigma_{g}\right)$ for all genus- $g$ surfaces, plus the chiral central charge, can fully characterize $2 d$ topological orders.

\section{ACKNOWLEDGMENTS}

L.K. was supported by the Science, Technology and Innovation Commission of Shenzhen Municipality (Grant No. ZDSYS20170303165926217), Guangdong Provincial Key Laboratory (Grant No. 2019B121203002), and NSFC (Grant No. 11971219). X.-G.W. was partially supported by NSF (Grant No. DMS-1664412) and Simons Collaboration on Ultra-Quantum Matter, which is a grant from the Simons Foundation (Grant No. 651440).

\section{APPENDIX: PROBE AND MEASURE TOPOLOGICAL ORDERS; PATH INTEGRAL APPROACH}

In this Appendix, we will only consider bosonic systems. Bosonic topological orders can also be realized by path integral on triangulated space-time $M^{D}$. Here, we will discuss how to characterize topological orders using path integral approach and the resulting partition functions.

\section{Topological partition function}

If the path integral is described by a well-defined quantum field theory (such as those that can be regularized by a tensor network path integral) that has no long-range correlations, it will describe an anomaly-free topological order. But how do we determine which anomaly-free topological order that the path integral produces? How do we determine whether two path integrals give rise to the same anomaly-free topological order or not?

One universal way to characterize all the topological orders is via the partition function of the system. In general, a partition function on a closed space-time $M^{D}$ may have a form

$$
Z\left(M^{D}\right)=e^{-c_{D} L^{D}-c_{D-1} L^{D-1}-\cdots-c_{0} L^{0}-c_{-1} L^{-1}-\cdots},
$$

where $L$ is the linear size of $M^{D}$. If the ground state does not contain pointlike defects, then $c_{1}=0$ since there is no world line in space-time. Similarly, if the ground state does not contain stringlike, membranelike, etc. defects, then $c_{2}=$ $c_{3}=\cdots=c_{d-1}=0$. In this case, we can define a volumeindependent partition function via

$$
Z^{\text {top }}\left(M^{D}\right) \equiv \lim _{L \rightarrow \infty} \frac{Z\left(M^{D}\right)}{e^{-c_{D} L^{D}}}=e^{-c_{0} L^{0}}
$$

When the calculated volume-independent partition function vanishes, it does not mean the partition function vanishes. It just means that $c_{i}>0$, for some $0<i<D$. This implies that the given space-time topology $M^{D}$ must contain pointlike, stringlike, etc. topological excitations.

We like to remark that it is not yet proven that using the above procedure to define volume-independent partition function $Z^{\text {top }}\left(M^{D}\right)$ always works. The assumption that $Z^{\text {top }}\left(M^{D}\right)$ can be well-defined equivalent to the assumption that the partition function of a topological quantum field theory can be defined via a microscopic path integral calculation. However, in Ref. [39] it is shown that only some ratios of $Z^{\text {top }}\left(M^{D}\right)$ are well defined. For example we may choose triangulated manifold, which contain four disjoint pieces: $\tilde{M}=M_{U} \sqcup$ $M_{D} \sqcup N_{U} \sqcup N_{D}$, where the boundaries of $M_{U}, M_{D}, N_{U}$, and $N_{D}$ are simplicial complexes all isomorphic to $B$. Gluing the boundaries in different ways gives rise to $M_{1}=\left(\begin{array}{c}M_{U} \\ M_{D}\end{array} N_{U} N_{D}\right.$

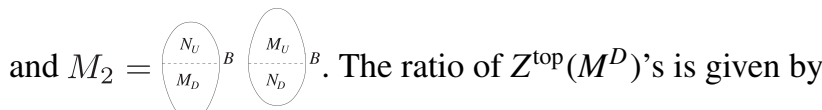

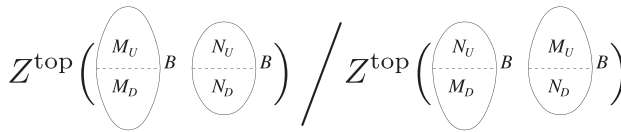

$$
\begin{aligned}
& =Z\left(\begin{array}{l}
M_{U} \\
M_{D}
\end{array} N_{U} N_{D}\right) / Z\left(\begin{array}{l}
N_{U} \\
M_{D}
\end{array} M_{U} N^{B}\right) \\
& =\frac{Z\left(M_{U} \cup M_{D}\right) Z\left(N_{U} \cup N_{D}\right)}{Z\left(N_{U} \cup M_{D}\right) Z\left(M_{U} \cup N_{D}\right)}
\end{aligned}
$$

since the volume term cancel exactly. Such ratios are topological invariants.

\section{Gravitational Chern-Simons term and winding numbers}

To use partition function to characterize topological order, we consider volume-independent partition function $Z^{\text {top }}\left(M^{D}\right)$ on closed space-time manifolds $M^{D}$. To understand the universal structures in the partition functions, let us use $\mathcal{M}_{M^{D}}$ to denote the moduli space of the closed space-time $M^{D}$ with different metrics but the same topology. Then the partition function $Z^{\text {top }}(-)$ can be viewed as a map from $\mathcal{M}_{M^{D}}$ to $\mathcal{C}$.

Claim 6. If a path integral describes a short-range correlated systems, then its volume-independent partition function on the moduli space $\mathcal{M}_{M^{D}}$ of a closed space-time $M^{D}$ is either always nonzero: $Z^{\text {top }}\left(M^{D}\right) \neq 0$, or always zero: $Z^{\text {top }}\left(M^{D}\right)=0$. 
Proof. If a volume-independent partition function is zero at some isolated points (or regions) of the moduli space $\mathcal{M}_{M^{D}}$, then a small local perturbation will make it nonzero. This will represent a diverging response, which should not occur for short-range correlated systems. Therefore, $Z^{\text {top }}\left(M^{D}\right)$ is either always nonzero or always zero.

So the nonzero volume-independent partition function $Z^{\text {top }}(\cdot)$ is actually a map $Z^{\text {top }}: \mathcal{M}_{M^{D}} \rightarrow \mathcal{C}-\{0\} \sim U(1)$. If $\pi_{1}\left(\mathcal{M}_{M^{D}}\right) \neq 0$, such map may have a nontrivial winding number.

Since $\pi_{1}\left(\mathcal{M}_{M^{D}}\right)=\operatorname{MCG}\left(M^{D}\right)$, the winding number is a group homomorphism $\operatorname{MCG}\left(M^{D}\right) \rightarrow \mathcal{Z}=\pi_{1}(U(1))$. So the winding numbers (i.e., the group homomorphisms) always form integer classes $\mathcal{Z}$. This leads us to believe that the winding numbers [or the group homomorphism $\operatorname{MCG}\left(M^{D}\right) \rightarrow \mathcal{Z}$ ] are always realized by the partition function $Z^{\text {top }}\left(M^{D}\right)$ that contains the gravitational Chern-Simons term $\omega_{D}$

$$
Z^{\text {top }}\left(M^{D}\right) \sim e^{i \int_{M^{D}} \omega_{D}},
$$

where the gravitation Chern-Simons term $\omega_{D}$ is given by

$$
d \omega_{D}=\sum \kappa_{n_{1} n_{2} \ldots} P_{n_{1} n_{2} \ldots},
$$

and $P_{n_{1} n_{2} \ldots}$ is a combination of Pontryagin classes, which are the only integer characteristic classes of oriented manifolds. Noticing that the winding numbers are given by $\frac{1}{2 \pi} \int_{M^{D} \curlywedge_{f} S^{1}} d \omega_{D}$, we have:

Claim 7.

$$
\int_{M^{D} \curlywedge_{f} S^{1}} \frac{d \omega_{D}}{2 \pi}=\sum \frac{\kappa_{n_{1} n_{2} \cdots}}{2 \pi} \int_{M^{D} \curlywedge_{f} S^{1}} P_{n_{1} n_{2} \cdots} \in \mathcal{Z}
$$

for any mapping torus $M^{D} \curlywedge_{f} S^{1}$ where $Z^{\text {top }}\left(M^{D}\right)$ is nonzero.

Such type of winding numbers and the partition function exist only when the space-time dimension $D=4 k+3$, since the gravitational Chern-Simons term exists only in $D=4 k+$ 3 space-time dimensions. We also note that there is always one combination of Pontryagin classes for each $D=4 k+3$ (corresponding to the signature $\sigma$ of the manifold), whose value on mapping torus is always zero (see Corollary 1.0.6. in Ref. [40]). For such Pontryagin class, the corresponding gravitational Chern-Simons term $\omega_{D}^{\sigma}$ can have an unquantized coefficient. For example, in $2+1$ dimensions, the naive consideration of diffeomorphism invariance appear to require the gravitational Chern-Simons term $\omega_{3}$ to have a coefficient that is quantized as $\kappa=\frac{2 \pi c}{24}, c=0 \bmod 8$. However, the above more careful consideration indicates that $c$ does not have to be quantized as $c=0 \bmod 8$. This is consistent with the well-known fact that there are many $2+1 \mathrm{D}$ anomaly-free topological orders with $c$ different from $0 \bmod 8$, despite $c$ must satisfy certain conditions (see Claims 3 and 3 ).

Clearly, two bosonic systems that give rise to partition functions with different winding numbers must belong to two different phases. So the winding numbers of partition functions are a type of topological invariants that can be used to probe and measure the anomaly-free topological orders.

\section{Beyond winding numbers}

To have an example of topological orders with nonzero winding numbers, we note that invertible topological orders are described by volume-independent partition functions that are pure $U(1)$ phase.

Claim 8. Since it has only trivial excitations, the volumeindependent partition function of an invertible topological order is nonzero for any closed space-time manifold $M^{D}$.

In particular the $\mathcal{Z}$ class of invertible topological order (such as the $E_{8}$ quantum Hall state in $D=3$ ) are described by

$$
Z^{\text {top }}\left(M^{D}\right)=e^{i 2 \pi \int_{M^{D}} \omega_{D}}
$$

We have shown that $\kappa_{n_{1} n_{2} \ldots}$ is quantized [see Eq. (A5)] if $P_{n_{1} n_{2} \ldots}$ is nonzero on some mapping torus.

However, for invertible topological orders, $\kappa_{n_{1} n_{2} \ldots}$ can be quantized even if $P_{n_{1} n_{2} \ldots}$ is zero on any mapping torus. To see this, we need to consider more general loop, i.e., more general mapping torus, where the topology of the fiber $M^{d}$ can change as we go along the loop. In this case, the more general mapping torus can be any closed $(D+1)$-dimensional manifold $M^{D+1}$. Thus we require that:

Claim 9. For invertible bosonic topological orders

$$
e^{i 2 \pi \int_{M^{D+1}} d \omega_{D}}=e^{i 2 \pi \sum \kappa_{n_{1} n_{2} \cdots} \int_{M^{D+1}} P_{n_{1} n_{2} \cdots}}=1
$$

for any closed $M^{D+1}$, which leads to a quantization of $\kappa_{n_{1} n_{2} \ldots}$.

We note that even $\omega_{D}^{\sigma}$ is required to have a quantize coefficient. For example, for $2+1 \mathrm{D}$ invertible topological orders

$$
\begin{aligned}
Z^{\mathrm{top}}\left(M^{3}\right) & =e^{i \kappa \int_{M^{3}} \omega_{3}}=e^{i \frac{2 \pi c}{24} \int_{M^{3}} \omega_{3}} \\
& =e^{i \frac{2 \pi c}{24} \int_{M^{4}} p_{1}}, \quad \partial M^{4}=M^{3},
\end{aligned}
$$

where $c \equiv 12 \kappa / \pi$ must be quantized as $0 \bmod 8$, since $\int_{N^{4}} p_{1}=0 \bmod 3$ for closed four-manifold $N^{4}$. In fact $c$ is the chiral central charge of the edge states and the above partition function describes the stacking of $c / 8 E_{8}$ quantum Hall states.

\section{Probing and measuring the anomaly-free topological orders}

In the previous section, we have discussed the quantization of $\kappa_{n_{1} n_{2} \ldots}$ for invertible topological orders. For noninvertible topological orders, we also have gravitational Chern-Simons

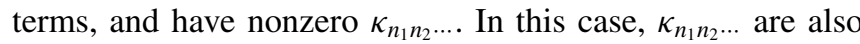
quantized. However, the quantization condition are weaker, since the volume-independent partition function is nonzero only on some subclass of closed manifolds. For example, for bosonic topological orders with emergent fermions, the volume-independent partition function vanishes on spacetime that is not spin. The volume-independent partition function can be nonzero only on spin manifolds. Only the spin manifolds can impose the quantization conditions on

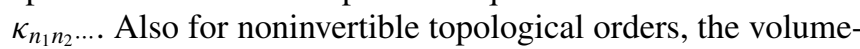
independent partition function is not just a phase factor.

Claim 9. The noninvertible topological orders are characterized by the following topological invariants:

(i) Quantized gravitational Chern-Simons terms (i.e., quantized $\kappa_{n_{1} n_{2} \ldots}$ 's)

(ii) The absolution values of volume-independent partition function $\left|Z^{\text {top }}\left(M^{D}\right)\right|$, on space-time $M^{D}$ with vanishing Euler and Pontryagin numbers (i.e., $\left|Z^{\mathrm{top}}\left(M^{D}\right)\right|$ is a constant on moduli space $\mathcal{M}_{M^{D}}$ ). 


\section{How to probe and measure the boundary-gappable topological orders}

We know that a boundary-gappable topological order can be described by a topological path integral that is independent of retriangulation of space-time and independent of local change of space-time metrics. The topological path integral directly give rise to the volume-independent partition function $Z^{\text {top }}\left(M^{D}\right)$, which is constant on $\mathcal{M}_{M^{D}}$ locally. Such a topological path integral is a fixed point of the renormalization group transformation. We propose that:

Claim 10. For boundary-gappable topological orders, we can use the volume-independent partition function $Z^{\text {top }}\left(M^{D}\right)$ of topological path integral to probe and measure them.

This conjecture has lead to some related research and is confirmed for simple boundary-gappable topological orders [29-31]. Since the topological path integral is retriangulation invariant, we see that $Z^{\text {top }}\left(M^{D}\right)$ is not only independent of volume, it is also independent of shape. It only depends on the topology of $M^{D}$. Therefore, the volume-independent partition function $Z^{\text {top }}\left(M^{D}\right)$ is a topological invariant for $D$ manifold $M^{D}$. It might be even true that different boundary-gappable topological orders give different topological invariants for at least some $M^{D}$ 's. In $2+1$ dimensions, the topological invariants from boundary-gappable topological orders are the Turaev-Viro invariants for three-manifolds [27].

We like to remark that the relation between volumeindependent partition functions $Z_{\mathrm{top}}\left(M^{D}\right)$ and boundarygappable topological orders is not one to one. Two volumeindependent partition functions $Z_{\text {top }}\left(M^{D}\right)$ differ by a factor $W^{\chi\left(M^{D}\right)} e^{i \sum_{\left\{n_{i}\right\}} \phi_{n_{1} n_{2} \cdots} \int_{M^{D}} P_{n_{1} n_{2} \cdots}}$ actually describe the same topological order. Here $W$ is a constant. This is because the factor $W^{\chi\left(M^{D}\right)} e^{i \sum_{\left\{n_{i}\right\}} \phi_{n_{1} n_{2} \cdots} \int_{M^{D}} P_{n_{1} n_{2} \cdots}}$ can be produced by local counterterms, which are deformations within the same phase.

\section{Applications}

For bosonic 2+1D invertible topological orders, its volume-independent partitions is nonzero in any closed orientable manifold $M^{3}$. From the quantization $\int_{M}^{4} p_{1}=0 \bmod 3$, we find that the chiral central charge $c=0 \bmod 8$. For $2+1 \mathrm{D}$ noninvertible topological orders with emergent fermions, its volume-independent partitions must be zero on any closed orientable manifold $M^{3}$ that is not spin. From the quantization $\int_{M^{4}} p_{1}=0 \bmod 48$ for any spin manifold $M^{4}$, we see that $c$ only need to satisfy $2 c n=0 \bmod 1$ for a certain set of integers $n$. Since the partition function may not nonzero for all spin manifold, we cannot conclude $2 c n=0 \bmod$ 1 for all integers.

The simplest class of $2+1 \mathrm{D}$ bosonic topological orders with emergent fermion has quantum dimensions $\left(d_{i}\right)=$ $(1,1, \sqrt{2})$. Their chiral central charge is given by $c=\frac{2 k+1}{2}$, $k \in \mathcal{Z}$. We believe that the partition functions for those topological orders are nonzero for any $2+1 \mathrm{D}$ spin manifolds since those topological orders can be obtained by gauging the fermion-number-parity in $p+i p$ superconductors in $2+1$ dimensions. So the chiral central charge for those bosonic topological orders must satisfy $2 c n=0 \mathrm{mod}$ 1 for all integers $n$, i.e., $2 c=0 \bmod 1$. This is consistent with $c=\frac{2 k+1}{2}$.

There is a $2+1 \mathrm{D}$ topological order whose edge states are described SU(2) level 6 conformal field theory with chiral central charge $c=\frac{9}{4}$. Such a topological order also has an emergent fermion. Since the chiral central charge does not satisfy $2 c=0 \bmod 1$, the volume-independent partition functions for such a topological order cannot be nonzero on all spin manifolds. It is an open problem to understand on which class of manifolds that the volume-independent partition functions are nonzero.
[1] V. Kalmeyer and R. B. Laughlin, Phys. Rev. Lett. 59, 2095 (1987).

[2] X. G. Wen, F. Wilczek, and A. Zee, Phys. Rev. B 39, 11413 (1989).

[3] X. G. Wen, Phys. Rev. B 40, 7387 (1989).

[4] X. G. Wen and Q. Niu, Phys. Rev. B 41, 9377 (1990).

[5] X. G. Wen, Int. J. Mod. Phys. B 04, 239 (1990).

[6] E. Keski-Vakkuri and X.-G. Wen, Int. J. Mod. Phys. B 07, 4227 (1993).

[7] E. Witten, Commun. Math. Phys. 121, 351 (1989).

[8] A. G. Abanov and A. Gromov, Phys. Rev. B 90, 014435 (2014).

[9] A. Gromov, G. Y. Cho, Y. You, A. G. Abanov, and E. Fradkin, Phys. Rev. Lett. 114, 016805 (2015).

[10] X. G. Wen, Phys. Rev. B 41, 12838 (1990).

[11] C. L. Kane and M. P. A. Fisher, Phys. Rev. B 55, 15832 (1997).

[12] A. Hatcher, Vector Bundles and K-Theory, version 2.2, 2017,

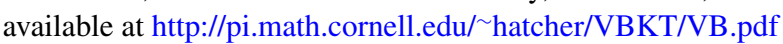

[13] X.-G. Wen, Phys. Rev. D 88, 045013 (2013).

[14] L. Kong and X.-G. Wen, arXiv:1405.5858.

[15] T. Church, B. Farb, and M. Thibault, J. Topol. 5, 575 (2012).

[16] S. Galatius, I. Madsen, and U. Tillmann, J. Am. Math. Soc. 19, 759 (2007).
[17] J. F. Ebert, arXiv:math/0611612.

[18] H. Endo, Osaka J. Math. 35, 915 (1998).

[19] C. Vafa, Phys. Lett. B 206, 421 (1988).

[20] G. Anderson and G. Moore, Commun. Math. Phys. 117, 441 (1988).

[21] M. Barkeshli and X.-G. Wen, Phys. Rev. B 79, 195132 (2009).

[22] X.-G. Wen, Nat. Sci. Rev. 3, 68 (2015).

[23] T. Lan, L. Kong, and X.-G. Wen, Phys. Rev. B 94, 155113 (2016).

[24] P. Putrov, J. Wang, and S.-T. Yau, Ann. Phys. (NY) 384, 254 (2017).

[25] J. Wang, K. Ohmori, P. Putrov, Y. Zheng, Z. Wan, M. Guo, H. Lin, P. Gao, and S.-T. Yau, Prog. Theor. Exp. Phys. 2018, 053A01 (2018).

[26] M. A. Levin and X.-G. Wen, Phys. Rev. B 71, 045110 (2005).

[27] V. Turaev and O. Viro, Topology 31, 865 (1992).

[28] S. X. Cui, Quantum Topology 10, 593 (2019).

[29] L.-Y. Hung and X.-G. Wen, Phys. Rev. B 89, 075121 (2014).

[30] H. Moradi and X.-G. Wen, Phys. Rev. Lett. 115, 036802 (2015).

[31] H. He, H. Moradi, and X.-G. Wen, Phys. Rev. B 90, 205114 (2014). 
[32] Y. Zhang, T. Grover, A. Turner, M. Oshikawa, and A. Vishwanath, Phys. Rev. B 85, 235151 (2012).

[33] H.-H. Tu, Y. Zhang, and X.-L. Qi, Phys. Rev. B 88, 195412 (2013).

[34] M. P. Zaletel, R. S. K. Mong, and F. Pollmann, Phys. Rev. Lett. 110, 236801 (2013).

[35] L. Cincio and G. Vidal, Phys. Rev. Lett. 110, 067208 (2013).
[36] E. Rowell, R. Stong, and Z. Wang, Commun. Math. Phys. 292, 343 (2009).

[37] M. Mignard and P. Schauenburg, arXiv:1708.02796.

[38] X. Wen and X.-G. Wen, arXiv:1908.10381.

[39] X.-G. Wen and Z. Wang, Phys. Rev. Res. 2, 033030 (2020).

[40] J. Ebert, J. Reine Angew. Math. 2013, 1 (2013). 